\title{
AMON: A Wearable Multiparameter Medical Monitoring and Alert System
}

\author{
U. Anliker, Member IEEE, J.A. Ward, Member IEEE, P. Lukowicz, Member IEEE, G. Tröster, Member IEEE, F. \\ Dolveck, M. Baer, F. Keita, E. Schenker, F. Catarsi, Member IEEE, L. Coluccini, A. Belardinelli, D. Shklarski, \\ M. Alon, E. Hirt, Member IEEE, R. Schmid, and M. Vuskovic.
}

\begin{abstract}
This paper describes AMON, a wearable medical monitoring and alert system targeting high-risk cardiac/respiratory patients. The system includes continuous collection and evaluation of multiple vital signs, intelligent multiparameter medical emergency detection, and a cellular connection to a medical centre. By integrating the whole system in an unobtrusive, wrist-worn enclosure and applying aggressive low power design techniques, continuous, long-term monitoring can be performed without interfering with the patients everyday activities and without restricting their mobility.

In the first two and a half years of this EU IST sponsored project, the AMON consortium has designed, implemented and tested the described wrist worn device, a communication link, and a comprehensive medical centre software package.

The performance of the system has been validated by a medical study with a set of 33 subjects.

The paper describes the main concepts behind the AMON system and presents details of the individual subsystems and solutions as well as the results of the medical validation.
\end{abstract}

Index Terms - multiparameter, wrist-worn, wearable, medical device, telemedicine, validation.

\section{INTRODUCTION}

Wearable systems can be broadly defined as mobile electronic devices that can be unobtrusively embedded in the user's outfit as part of the clothing or an accessory. In particular, unlike conventional mobile systems, they can be operational and accessed without or with very little hindrance to user activity [1], [2]. Today, wearable systems range from microsensors, seamlessly integrated in textiles through consumer electronics, embedded in fashionable clothes and computerized watches to belt-worn PCs with a head-mounted display.

One obvious application of wearable systems is the monitoring of physiological parameters in a mobile environment. In this context, devices targeting the sport and recreational market have been very successful. However, the majority of such recreational devices are not suitable for medical monitoring of high-risk patients. Those devices that have been qualified

Manuscript received August 30, 2003; revised February 17, 2004

Anliker, Ward, Lukowicz and Tröster are with the Wearable Lab ETH Zurich, Switzerland

Dolveck, Baer, and Keita are with SAMU 92, Hopital Raymond Poincare 92380 Garches, France

Schenker is with MDirect, Israel

Catarsi and Coluccini are with Aurelia Microelettronica, Italy

Belardinelli is with Institute of Clinical Physiology - CNR - National Research Council, Pisa, Italy

Shklarski and Alon are with Tadiran Spectralink, Israel

Hirt, Schmid and Vuskovic are with Art of Technology, Switzerland for medical use are usually fairly simple measuring just one or two parameters and providing little or no online analysis.

The AMON (Advanced care and alert portable telemedical MONitor) project - financed by the EU FP5 IST program - takes this idea a step further, aiming at continuous medical monitoring for high-risk cardiac/respiratory patients. This includes continuous collection and evaluation of multiple vital signs, intelligent multi-parameter medical emergency detection, and a cellular connection to a telemedicine centre. The idea is that by using an unobtrusive, wrist-worn device the monitoring can be performed without interfering with the patients everyday activities and without restricting their mobility. Thus, people currently confined to hospital or their homes can lead normal lives while knowing that any medical problems will be detected in time and help will be dispatched. Additionally, physicians are provided with a greater level of information about a patient's condition - from a natural setting - thus enabling them to better tailor treatment.

Mobile monitoring of physiological parameters has been studied by many research groups, e.g. [3], [4]. For data acquisition, personal Holter monitors are often used, e.g. for ECG and blood pressure.

For one or two parameter measuring there are several devices commercially available: Agilent, Phillips and Nellcor all produce handheld pulse oximeters for non-invasive monitoring of blood oxygen saturation and pulse (SpO2). For blood pressure, Omron produce a range of portable wrist devices [5].

For the wellness and lifestyle market there are several products. The chest-worn Polar can measure heart rate, but provides no further information about ECG, such as QRS or QT [6]. The SenseWear from BodyMedia measures activity, temperature parameters and galvanic skin response. An additional chestworn sensor for heart rate measurement can be attached [7].

The University of Alabama has developed a wearable ECG monitor with real time feedback to the user [8]. Other sensors can be integrated using a wireless body area network of intelligent sensors [9], but the system contains multiple sensors that are not integrated into a single device. Other multiparameter logging devices, such as Escort Guardian [10] or Micropaq [11], are also not integrated and have only standard configuration.

Several research groups have developed portable medical devices for home care, these transmit measurements without analysis to a telemedicine centre [12], [13] and [14]. Other groups use monitoring devices to understand the reaction of 
the human body to stress [15] or to improve recovery process e.g. from strokes [16]. However, the analysis performed by these is often performed offline at the telemedicine centre.

Compared to the above mentioned projects the AMON system has several unique features:

1) Multiparameter Monitoring: The AMON system is capable of measuring blood pressure, $\mathrm{SpO} 2$ and a one lead ECG, all in a single device. It has an interface to additional external sensors including a full 12 lead ECG. At time of writing, there are currently no handheld or portable devices which combine all of these measurements.

2) Activity Recognition: Using a 2 axis acceleration sensor integrated in the system, AMON is capable of detecting the level of user activity and correlating it with the vital signs.

3) Online Analysis and Emergency Detection: The wristworn device can perform an analysis of all measurements online, presenting them in appropriate form to both wearer and remote telemedicine centre. For emergency detection the analysis can incorporate patients profile and activity information to reduce the number of false alarms.

4) Flexible Communication Interface: The cellular connection to the telemedicine centre features a flexible communication channel that can use a direct connection as well as SMS (short message system) services. It can also be easily extended to incorporate a TCP/IP based link.

5) All-in-One Wrist-Worn System: The AMON system combines all sensors, communication and processing devices in a single, ergonomic wrist-worn enclosure. The integration in one device has the disadvantage of making the signal acquisition, in particular pulse and ECG, harder as compared to distributed systems that place sensors (e.g. ECG electrodes) on several specific body locations. However, for the envisioned target group and application such 'all-in-one design' is essential. It must be assumed that many in the target group are technology averse and possibly have some cognitive impairment (e.g. forgetfulness). On the other hand the system must be worn on a daily basis and be put on without assistance. Thus, it is not acceptable to have the user put on multiple devices, at different, exactly defined body locations.

The integration of all components in a wrist-worn enclosure and the derivation of vital signs from wrist-based sensors is one of the main contributions of AMON.

In order to address the potential for AMON as a medical device - as opposed to merely a lifestyle product - a clinical validation was performed. Although the first AMON prototypes have varying degrees of success in achieving medical accuracy on all measurement combinations, our results clearly demonstrate the feasibility of the concept and solutions to the key technological and scientific issues.

The remainder of the paper is divided as follows: the next section (II) gives an overview of the system; section III

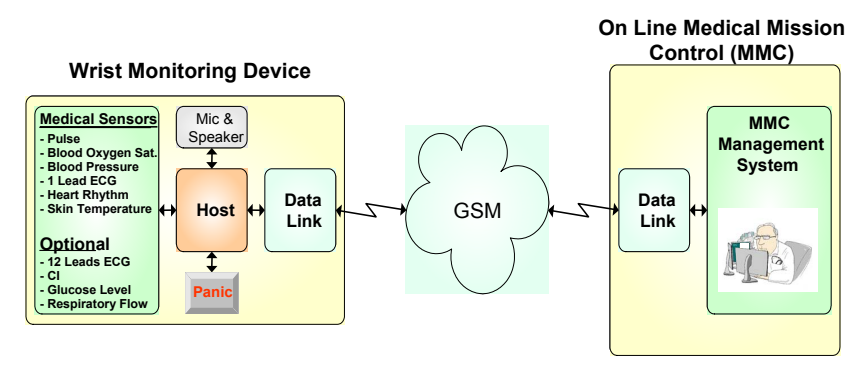

Fig. 1. System overview: AMON; Wrist Worn Medical device with GSM/UMTS link to the telemedicine centre.

describes the sensor system and the processing algorithms; section IV describes the data processing and communication hardware in the wrist unit and at the telemedicine centre. We then present the results of the first medical trials and our conclusions in sections $\mathrm{V}$ and VI.

\section{SySTEM OVERVIEW}

The AMON System comprises two separate parts: a wristworn unit and a stationary unit at the telemedicine centre [Figure 1].

Key innovations of the wrist-worn part are: the integration of multiple medical monitors and evaluation software in a single device; the non-standard placement of measurement sensors; and miniaturisation to a level where the device is wearable at the wrist. In addition mobile communication capability is integrated in the device in form of an on-board GSM transceiver. The tranceiver enables data exchange with the medical centre.

At the telemedicine centre, incoming medical data from wrist-worn units is collected and processed by trained medical personnel. This side of the system is based on a JAVA server platform and a windows workstation connected to a GSM/UMTS transceiver. The software allows authorised personnel to securely access medical records and details of a patient in addition to the incoming data from the wrist-worn units. It allows measurement requests to be made direct from the telemedicine centre; additionally, provision is made for direct communication with patients.

AMON monitors pulse, oxygen saturation ( $\mathrm{SpO} 2)$, skin temperature and activity via acceleration continuously. Continuously in our medical application means for $\mathrm{SpO} 2$ thirty seconds of measurement every two minutes and every two minutes a temperature reading. Blood pressure and one lead ECG (thirty seconds of raw data) is measured three times a day or on request by the user or the telemedicine centre. The data is analysed on-device after every measurement. The analysis includes all digital signal processing needs for signal enhancement (e.g. filtering), all computation required to convert the measured values into medical values, and initial evaluation of those values.

The main conversion algorithms [see section III] includes the computation of blood pressure from the pressure sensor signal and the derivation of the QRS width, RR distance and QT intervall from the ECG signal. 
The initial analysis starts with a comparison of the pulse and oxygen saturation with predefined, patient specific values. Based on the results of this analysis, three different scenarios are possible:

- Everything ok: Wearer is informed that all measurements are fine.

- Parameter out of range: A re-measurement is performed. If the outcome is the same as before, the user is informed and additional measurements are required. The wristworn device determines the type and initiates the measurement. The type of measurement includes $\mathrm{SpO} 2$, blood pressure and ECG. Taking into account combined results of all measurements, the system then decides whether to alert the telemedicine centre or not.

- More than one parameter out of range: The data is automatically sent to the telemedicine centre, where it triggers an alarm. The medical personnel then performs a detailed analysis of all data re-checking the automatic evaluation. Depending on telemedicine centre analysis of this data, further steps are taken: in a non-life threatening emergency situation, perhaps requiring modification to treatment or further tests, the patient is referred to his usual physician; if a life threatening situation is detected, the telemedicine centre can immediately organise prehospital care and transportation.

In normal mode - everything ok - the data (raw data and extracted medical values) is transmitted to the telemedicine centre three times a day. The centre will re-check the measurements; the higher computation power and storage capacity afforded by the telemedicine centre is used for more powerful data analysis [see section IV-C].

In all cases, the patient is informed as to their own status and that of the device. In the event of a failure to initiate communication with the telemedicine centre, the user is informed appropriately. In non-critical situations, the results can be stored (up to four days of data) on-device and sent at such a time as communication is restored. The device will periodically attempt to regain communication throughout any down time.

The telemedicine centre should be able to cope with multiple remote devices simultaneously. In the event of an abnormal reading, communication should be immediate. Of course this is subject to availability of the GSM connection, and for this reason users of the system should be instructed, prior to being given the device, to remain within areas with good coverage.

\section{SENSORS AND AlgorithmS}

For the designated target group of cardiac and respiratory patients the key parameters that need to be monitored are pulse, oxygen saturation (SpO2), blood pressure and ECG. Additionally, it is advantageous to have some information about the level of physical activity. This section describes the sensors and the signal processing algorithms used in the AMON system to derive the above information.

The main challenges faced by the design at this stage was the ability to derive all the above information from wrist-worn sensors only and the need to keep the size and the power consumption to a minimum.

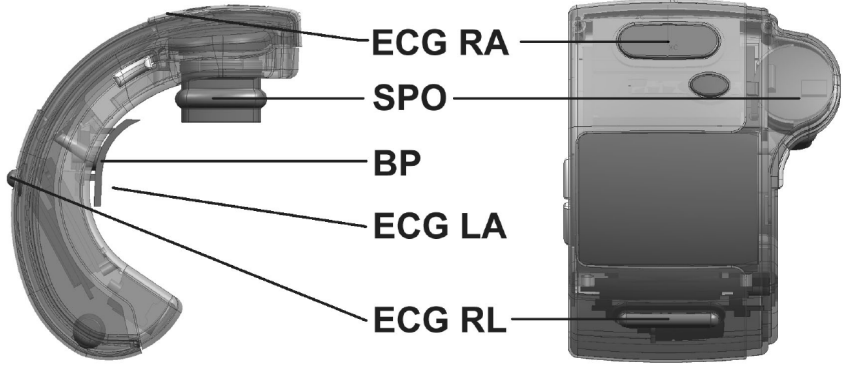

Fig. 2. AMON Sensors: SpO2, one lead ECG (RA, LA, RL), Blood pressure (pressure, pump and valve), acceleration (not shown) and temperature (not shown)

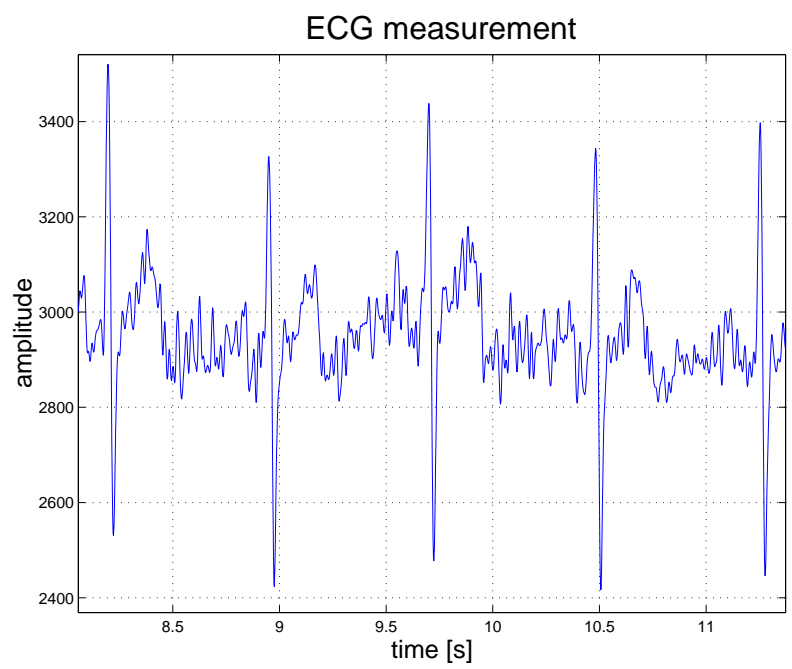

Fig. 3. AMON one lead ECG sample measurement: a digital bandpass filter [0.015 40$] \mathrm{Hz}$ has been applied to reduce noise. Heart rate, QT interval and QRS duration can be detected.

\section{A. SPO Sensor}

The measurement by pulse oximeter is based on the absorption of two wavelengths through a pulsatory arteriolar flow. Arterial saturation in oxygen and pulse are assessed by comparing the changes of each wavelength during one pulsation. The sensors are either clippers at the tip of fingers, or adhesive. There are also reflectance sensors that can be placed on the forehead in front of the temporal artery or that can be put on the sternum. The principle is the same and is based on the absorption of two wavelengths cast and reflected by an arteriolar flow [17], [18].

The AMON device contains a reflectance sensor placed on the top of the wrist [Figure 2]. It uses two wavelengths, one at 660 nanometer and the other at 880 nanometer. A light detector harnesses the reflected waves. The pulse oximeter probe and signal processing algorithms have been developed and manufactured exclusively for the AMON project by SPO Medical Equipment Ltd, Israel [19] based on a specification by MDirect. 


\section{B. ECG Sensor}

To obtain an ECG the patient must be physically connected to a front-end amplifier with special bioelectrodes that convert ionic current flow of the body to the electron flow of the metallic wire. Silver-coated chest suction electrodes or adhesive silver/silver-chloride electrodes with chemical paste or gel can be used. The first prototype of AMON used silver/silver-chloride electrodes without paste or gel. During the first prototype testing the electrodes oxidised after two to three months and the input resistance raised to a level where no ECG signal could be measured. To eliminate oxidation, the second prototype used gold electrodes. The contact resistance is slightly higher, but a reasonable signal quality can be obtained [Figure 3].

1) Electrode Configuration: All electrodes are mounted in one wrist-worn device. These are placed as follows [Figure 2]: Left Arm (LA) electrode is inside the cuff, Right Arm (RA) electrode is on top; during a measurement the patient must touch the RA with his left hand. The Right Leg (RL) electrode is placed on top pointing to the wearer; during measurement this electrode must be in contact with the abdomen. In order to reduce common mode interference, a right leg drive circuit has been chosen [20] with gain set to 39 [21].

A high pass [cut off $0.05 \mathrm{~Hz}$ ] and a low pass [cut off $100 \mathrm{~Hz}$ ] filter is integrated in the amplifier stages. The signals are then digitised at $500 \mathrm{~Hz}$ sampling frequency.

2) Algorithm: The aim of the algorithm is to detect and measure a number of medical parameters from the ECG waveform, in particular: QRS complex width, RR distance and QT interval. A further desirable parameter, PR interval, was left out for this work due to the non-triviality of P-wave detection [22].

In keeping with the near real-time requirements and low processing power, a necessary requirement is that the algorithm is simple but accurate. The approach taken is based on a simplification of the QRS detection scheme of Pan and Tompkins [23]. This processes the sampled data as follows:

1) Differentiation - obtain information about signal slope.

2) Squaring the derivative - intensify frequency response curve of derivative.

3) Integrate over moving window - obtain information about slope and width of QRS complex, also filters out some unwanted spikes.

For QRS detection, a threshold set is computed during an initial learning stage (lasting 8 seconds): the upper threshold is calculated from $0.4 \mathrm{x}$ the average maximum on the integrated signal; from this, a lower threshold is calculated by another factor of 0.4. During the detection process, the current integrated moving window value is compared with the upper threshold. If this threshold is exceeded, an R-wave onset is assumed; QRS is confirmed by scanning backward (up to $100 \mathrm{~ms}$ ) for a dip below the lower-threshold. These threshold values are continually adjusted with each new QRS so as to compensate for variations in ECG baseline.

$\mathrm{R}$-wave is detected simply by searching for a peak in the raw signal following the detected QRS onset. Searching backwards for the closest negative slope from $\mathrm{R}$ obtains the Q-wave.

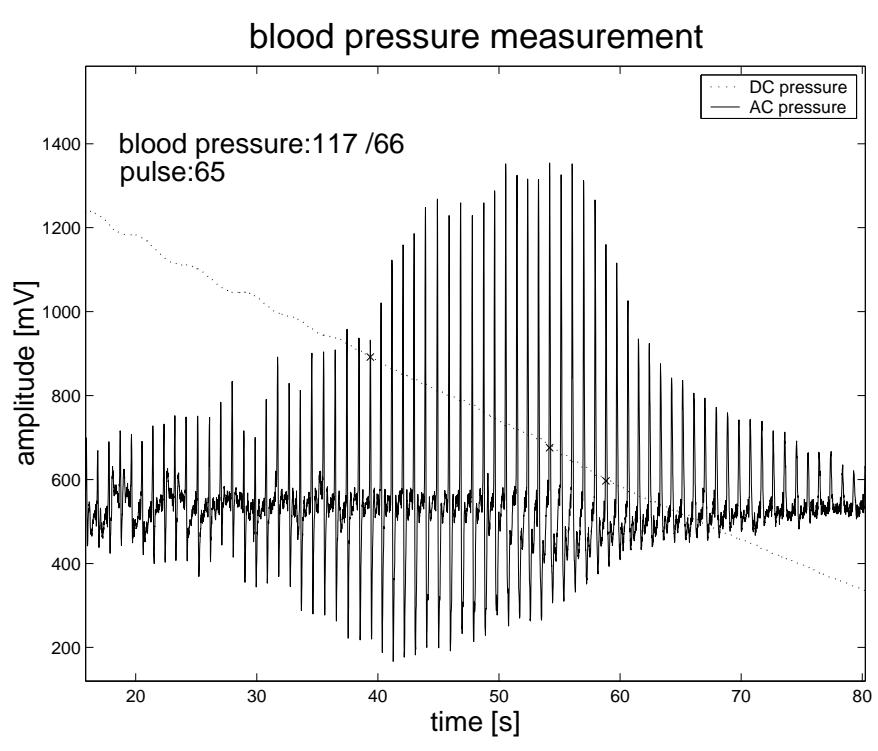

Fig. 4. AMON blood pressure sample measurement: standard oscillometric method is applied to calculate systolic and diastolic pressure; pulse measurement is done by peak detection in the AC signal.

Similarly the S-wave is found by searching a positive slope forwards of R. The maximum slope forward of $\mathrm{S}$ (for about $100 \mathrm{~ms}$ ) is denoted the T-onset. An estimation of the T-point is found simply by adding $80 \mathrm{~ms}$ to this.

The distances RR, QRS and QT are stored for every discovered QRS wave. For an overall result - as displayed to the user - averages are taken over all the valid QRS. Heart rate is calculated directly from RR.

\section{Blood Pressure}

1) Hardware and Algorithm Principles: The blood pressure measurements employ the standard oscillation method [24], [25] and [26]. The wrist and its vasculature are compressed by an encircling, inflatable compression cuff. The principle of the oscillometric method is a measurement of the amplitude of pressure change in the cuff as the cuff is inflated to about $30 \mathrm{mmHg}$ above systolic pressure and then deflated at a rate of $2-4 \mathrm{mmHg} / \mathrm{s}$. The oscillation signal varies from person to person, in general, it varies from less than $1 \mathrm{mmHg}$ to 4 $6 \mathrm{mmHg}$ [Figure 4]. For prototyping the cuff, pump and valve are taken from an OMRON device (model R5-I). Inflation and deflation rates are controlled by varying the power to the pump and valve using PWM (Pulse width modulated) signals.

When measuring blood pressure at the wrist, it is important that the wrist with the cuff is positioned at the same level as the heart. Best results are obtained when the left arm (with the device) is positioned at - but not holding - the shoulder.

The pressure signal is split into high frequency $(0.03 \mathrm{~Hz}$ to $30 \mathrm{~Hz})$ and low frequency parts $(0 \mathrm{~Hz}$ to $10 \mathrm{~Hz})$.

\section{2) Implementation:}

a) pressure: The blood pressure algorithm detects peaks and finds the maximum of the peaks in the high frequency part of the signal. To reduce noise, a small moving average window and later a curve approximation for the maximum 
region is applied. After removing the offset of $540 \mathrm{mV}$ in the high frequency signal, the standard algorithm of the oscillation method is applied.

b) pulse: The algorithm for pulse calculation is based on peak detection. The time difference between two peaks is averaged between systolic and diastolic pressure, this is then converted to peaks per minute.

\section{Acceleration Sensor}

Acceleration sensors provide information on the activities of the wearer. Three uses of this information are made: First, the pulse limits are set according to the activity level - e.g. walking, running or resting [27]. Second, a potentially dangerous fall could be detected [28], [29]. Finally a total lack of movement, combined with recent temperature and physiological sensor readings, can be used to indicate one of three possible scenarios: device has been removed from wearer - a constant, abnormal temperature and a complete lack of useful data from the sensors; wearer falls unconscious - a measurement was halted prematurely, earlier readings having indicated an emergency situation; rest or sleep - recent readings have not given reason to suspect the onset of an emergency situation.

In the later cases, it is acknowledged that any decisions regarding the device and wearer's state must be made by trained personnel at the telemedicine centre. The degree of movement should therefore be presented at the telemedicine centre as another parameter to be monitored alongside the physiological readings.

1) Activity detection: The AMON system requires only very simple activity analysis compared to other wearable activity detection applications, e.g. [30]. What we are interested in, is the level of physical activity without being able to distinguish specific actions. The main problem that our analysis has to deal with is the fact that intensive arm motion by itself is by no means an indication of strenuous physical activity. Thus for example eating, drinking or just talking and gesticulating involves arm motions that are not particularly strenuous. Our analysis is based on the fact that strenuous activity is mostly associated with (fast) walking or running. This in turn has a characteristic, periodic acceleration signature with the frequency indicating the walking speed (see Figure 5). This periodic signature can be detected even if the arms do not follow the walking motion directly through swinging and are engaged in some other activity.

\section{E. Temperature Sensor}

The temperature measurement has been included in the device for experimental purpose mostly. The consortium has intended to study if a reliable correlation between the sensor reading at the wrist and the core body temperature could be established. Unfortunately, this has not succeeded and the sensor cannot be used for medical purposes. The hand temperature is significantly below the body temperature, and there is no fixed relationship between the two. Temperature of the extremities change with ambient, health and physical conditions [31]. The sensor is therefore used only for functional testing and

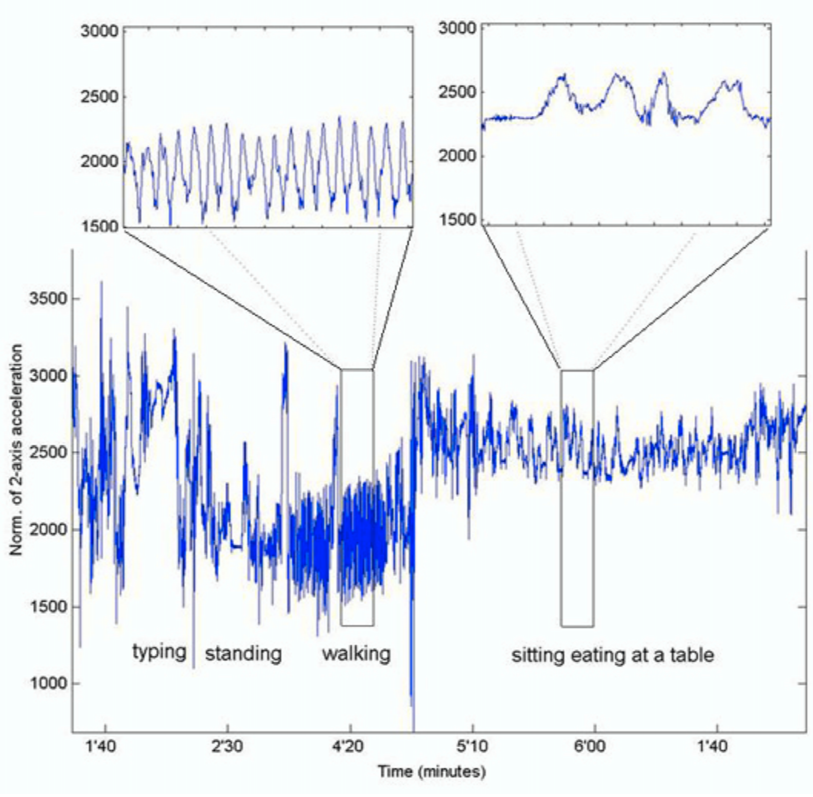

Fig. 5. Activity Readings: norm. of 2-axis acceleration data from the wrist during typical daily activities.

together with the acceleration sensor for verification if the device is being worn.

\section{F. High-Level Medical Algorithm}

The AMON high-level medical algorithms integrate data from different sensors on a single device. To our knowledge, no such algorithms are in clinical use today at home care set-up. All wrist medical algorithms are based on several sets of limits for vital signs. Based on comparison of these standard - or pre-customised - limits, the measurement results are assigned to one of five zones - normal, deviant (abnormal values), risk, high risk and error. The default medical ranges [see Table I], are set according to medical standard values, based on World Health Organisation (WHO) recommendations [32].

The wrist device has two customizable sets of parameters, which are set by the health care provider when handing the device over to the patient. The parameter values can be changed by the users physician, the care provider or in real time by the medical operator in the telemedicine centre via the cellular link.

The two sets represent a non-aerobic state and an aerobic state corresponding to the level of user activity. The parameters are set accordingly to age, gender and medical history. The selection of the active set is performed by user command or automatically by the wrist device when activity is detect.

The clinical algorithm follows five steps, this proceeds as follows:

- First Step: Comparison of measurement results to a range of values, and assignment to a risk zone accordingly.

- Second Step: Check the zone limits based on the average value derived from the last five results. Check erroneous measurements due to possible problem with the sensors. 
TABLE I

L1 AND H1 REPRESENT DEVIANT ZONE, L2 AND H2 RISK ZONE AND L3 AND H3 HIGH-RISK ZONE

\begin{tabular}{l|c|c|c|c|c|c|c} 
vital sign & L3 & L2 & L1 & Normal & H1 & H2 & H3 \\
\hline Systolic (mmHg) & $50-59$ & $60-79$ & $80-99$ & $100-130$ & $131-160$ & $161-200$ & $201-300$ \\
Diastolic (mmHg) & $40-44$ & $45-49$ & $50-59$ & $60-85$ & $86-90$ & $91-110$ & $111-140$ \\
SpO2 (\%) & $65-79$ & $80-91$ & $92-94$ & $95-100$ & & & 1001 \\
Pulse (per minute) & $40-44$ & $45-49$ & $50-59$ & $60-100$ & $101-120$ & $121-180$ & $181-250$ \\
QRS duration (s) & $0.01-0.03$ & & & $0.04-0.12$ & & & $0.121-0.35$
\end{tabular}

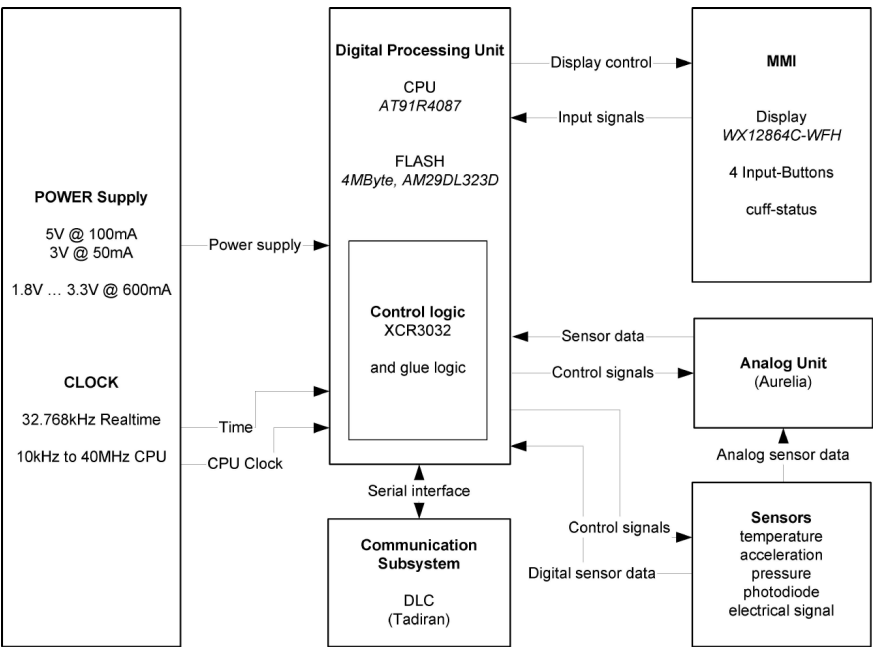

Fig. 6. Block diagram of the AMON wearable health monitoring device. Functional blocks: power supply, sensors, man machine interface (MMI), analog unit, digital processing unit and communication subsystem (DLW).

- Third Step: When previous steps indicate a risk or highrisk zone, determine if and what new measurement set is required.

- Fourth Step: Calculation of pulse based on two or three different measurements ( $\mathrm{SpO} 2$, blood pressure, and ECG). Each measurement is weighted according to its reliability.

- Fifth Step: Pattern recognition of the medical data for clinical diagnosis.

On each step a result is displayed and, if appropriate, sent to the telemedicine centre for further processing.

\section{SubSYSTEM IMPLEMENTATION}

\section{A. Wrist Device}

Figure 6 shows the five functional blocks of the wrist device: power supply, sensors, man machine interface (MMI), analog unit, digital processing unit and communication subsystem (DLC). The wrist device prototype weighs 286 grammes, the estimated run time is 2 days [see Table II] and includes 4MByte of flash and 136kByte on Chip SRAM for programme and data storage.

1) Low Power System Design: Battery life is one of the major design constrains in mobile healthcare systems. The system must be constantly operational without the need to frequently change or re-charge batteries. User expectation from widespread use of cell phones is that a device should run for at least a couple of days.
Several techniques are known from literature to reduce power consumption [33] such as Dynamic Voltage Scaling (DVS) [34] and Dynamic Power Management (DPM), this includes putting unused modules or components in sleep mode and clustering of communication.

Each functional module of AMON has different ways of reducing power consumption. The following paragraphs describe the applied low power techniques.

a) Power Supply: Several modules run at different voltages: digital parts $3 \mathrm{~V}$, CPU core voltage $1.8 \mathrm{~V}$ to $3.0 \mathrm{~V}$, analog module $5 \mathrm{~V}$, communication subsystem, pump and valve at battery voltage $(3.7 \mathrm{~V})$.

$5 \mathrm{~V}$ is generated by a step-up converter supplying the analog unit, the efficiency is $85 \%$ ( $1 \mathrm{~mA}$ to $50 \mathrm{~mA}$ output current). The efficiency drops to $10 \%$ or less if the power consumption is below $0.1 \mathrm{~mA}$. The step-up converter is only on during blood pressure, temperature, ECG and battery voltage readings. The influence of the ripple introduced by the switched converter is reduced by filtering and amplifier configuration.

Step-down regulators have higher efficiency than linear. The variable voltage $(1.8 \mathrm{~V}$ to $3.3 \mathrm{~V})$ is regulated by a step down converter at an efficiency of $93 \%(1 \mathrm{~mA}$ to $200 \mathrm{~mA})$. A digital potentiometer in the feedback path adjusts the output voltage for DVS. The digital 3V supply is obtained in a similar way.

b) Sensors: Sensors were selected with low power considerations in mind. Where feasible, passive sensors were chosen (temperature, ECG electrodes, pressure.)

The pulse oximeter was designed by SPO Medical Equipment Ltd. for low power, the datasheet reports $3.3 \mathrm{~mW}$ during measurement. Unfortunately, under test this is nearer $10 \mathrm{~mA}$ (an issue currently being resolved by the company concerned.)

c) Display: The power consumption of the display is $70 \mu \mathrm{A}$, backlight about $50 \mathrm{~mA}$. The backlight will be only activated by a button, and so be most of the time off.

d) Analog Unit: Special care for low power design was put in the analog processing unit. Originally it was foreseen to use a custom developed ASIC, this would have an expected subsystem consumption of less then $50 \mathrm{~mW}$. Using aggressive low power design technique a power consumption below $1 \mathrm{~mW}$ is possible [35]. Unfortunately the ASIC was not fully working by the project end; instead a discrete version, developed in parallel, was used for the prototypes. The consumption thus nears $120 \mathrm{~mW}$ [chapter IV-A.1.g].

e) Digital Processing Unit: For the most part, the wrist device shows time, pulse and oxygen saturation (SPO2), which is measured every two minutes for thirty seconds.

Although the SPO2 measurement is taken frequently, most of the calculation is performed using its own ASIC. The central CPU has no computation tasks and needs only to handle the 
TABLE II

POWER BUdGET OF THE WRIST DEVICE, DUTY CYCLE ARE BASED ON A TYPICAL DAY. BATTERY IS A 1.25AH LIION, CURRENT IS MEASURED FROM PROTOTYPES.

\begin{tabular}{l|r|r|r|r} 
function & current & voltage & duty cycle & current eff. \\
\hline GSM & $3.5 \mathrm{~mA}$ to $2.3 \mathrm{~A}$ & battery & $2.0 \%$ & $12.5 \mathrm{~mA}$ \\
Inflating & $220 \mathrm{~mA}$ & battery & $0.3 \%$ & $0.7 \mathrm{~mA}$ \\
Deflating & $90 \mathrm{~mA}$ & battery & $1.0 \%$ & $0.9 \mathrm{~mA}$ \\
Analog Unit & $40 \mathrm{~mA}$ & $5 \mathrm{~V}$ & $4.5 \%$ & $1.8 \mathrm{~mA}$ \\
Digital Unit & $9 \mathrm{~mA}$ & 1.8 to $3 \mathrm{~V}$ & $50 \%$ & $4.5 \mathrm{~mA}$ \\
SPO & $10 \mathrm{~mA}$ & $3 \mathrm{~V}$ & $50 \%$ & $5.0 \mathrm{~mA}$ \\
\hline Total & & & & $25.4 \mathrm{~mA}$
\end{tabular}

communication protocol, thus the load is low.

The AT91R40807 CPU allows a core voltage down to $1.8 \mathrm{~V}$ (at maximum $18 \mathrm{MHz}$ ), maximum operation frequency is $40 \mathrm{MHz}$ ( $3 \mathrm{~V}$ core Voltage). According to the datasheet, power consumption can be reduced from $135 \mathrm{~mW}$ (at $40 \mathrm{MHz}, 3.0 \mathrm{~V}$ ) to $22 \mathrm{~mW}(18 \mathrm{MHz}, 1.8 \mathrm{~V})$. The clock frequency can be reduced further, this reduces idle power consumption.

The computation load varies from 30MIPS during ECG and blood pressure measurements to a few commands every second for updating time or starting an SPO measurement. The use of Dynamic Voltage Scaling (DVS) would help to tune the computation capability according to these needs [36]. However, at time of writing, this has not yet been implemented for this project.

f) Communication Subsystem: The prototype uses a Siemens TC35 Cellular Engine. The subsystem is most of the time in sleep mode, this is still connected to the GSM network, and power consumption is $3 \mathrm{~mA}$. During data transmission/call the current can go up to $1.8 \mathrm{~A}$ peak, but will average at $300 \mathrm{~mA}$. In normal use, the link will be open for 10 minutes per day, to transmit $500 \mathrm{kB}$ of data.

g) Power Measurements: Table II gives an overview of the system power consumption. GSM, SPO and digital processing unit are the highest power consumers. The digital processing unit was measured at $3.0 \mathrm{~V}$ and $40 \mathrm{MHz}$.

Energy-wise, communication via wireless is expensive. More run time can be gained if data transmission is carried out when the network quality is high, thereby requiring less signal power.

SPO2 is measured for 30 seconds every two minutes. Our test showed that the sensor needs about 20 seconds to give stable results, thus it has a duty cycle of about $50 \%$.

The analog part is used during temperature, blood pressure and ECG measurement and is turned off most of the time (duty cycle of $4.5 \%$ ). The replacement of the discrete analog board with an ASIC will reduce the power consumption at least by a factor of 4 .

A blood pressure measurement takes about 20 seconds to inflate and 80 seconds to deflate the cuff. A duty cycle of $0.3 \%$ for inflating and $1.0 \%$ for deflating represents 10 measurements per day. Further improvements in the blood pressure algorithm can reduce the time for measurement, e.g. reduce end pressure of inflating or stop the measurements after diastolic pressure is passed.

\section{B. Communication}

The main task of the AMON communication system is to transmit reliable sensitive medical information through a secured channel from the AMON wrist device to the telemedicine centre and to handle requests/instructions from the centre to the user.

To implement the above requirements the communication protocol has been designed to support three types of communication channels:

1) SMS (Short Message Service) using the service provided by mobile phone network operators throughout Europe. This is used for messages with a small number of values, such as with the Blood Pressure results.

2) Virtual circuit switched communication channel established between the wrist device and the telemedicine centre through a mobile phone link. This is used for long messages such as a raw ECG data. It is essential in an emergency situation when a real time link is needed between the medical personnel and the patient's device.

3) An Internet based channel such as an FTP connection or custom TCP/IP-Stack. This has been foreseen to reduce the number of direct lines needed at the centre and provide more efficient international communication in future applications.

The communication protocol has been designed in such a way that all three channel types rely on the same message structure: header, message body and tail. The header includes information such as source and destination of the message and the type of content. The body contains the results of the medical measurements (processed results or raw data), and the tail enables error correction capabilities.

The communication system implements the requirements of European legislations regarding data privacy [37]. The legislation determines that respect for the patient's privacy should be guaranteed at all times during the processing and transmission of medical data. It is implemented by first removing any identity of the user from the data and then performing encryption and authentication (as part of the inherent GSM/GPRS protocol.)

The communication system consists of a controller that is responsible for all the above activities and of a cellular engine (GSM). From the cellular network point of view the AMON device acts in the same way as a regular cellular phone.

Since the mid-nineties research groups have been working on methods to calculate the position of a mobile device connected to a GSM network. So long as the serving cell is known, location accuracy of several hundred meters in urban regions can be obtained. Other methods to enhance the position quality are described in [38] and [39]. This will help to locate the wearer of AMON and to send an ambulance if needed.

\section{Telemedicine Centre}

The telemedicine centre (TMC) is based on a JAVA server platform running on a Windows PC with a GSM data link. The TMC facilitates, tracks, monitors and reports the patient condition. Medical data transmitted from the wrist-worn device is analysed by specialised software, designed by MDirect, 


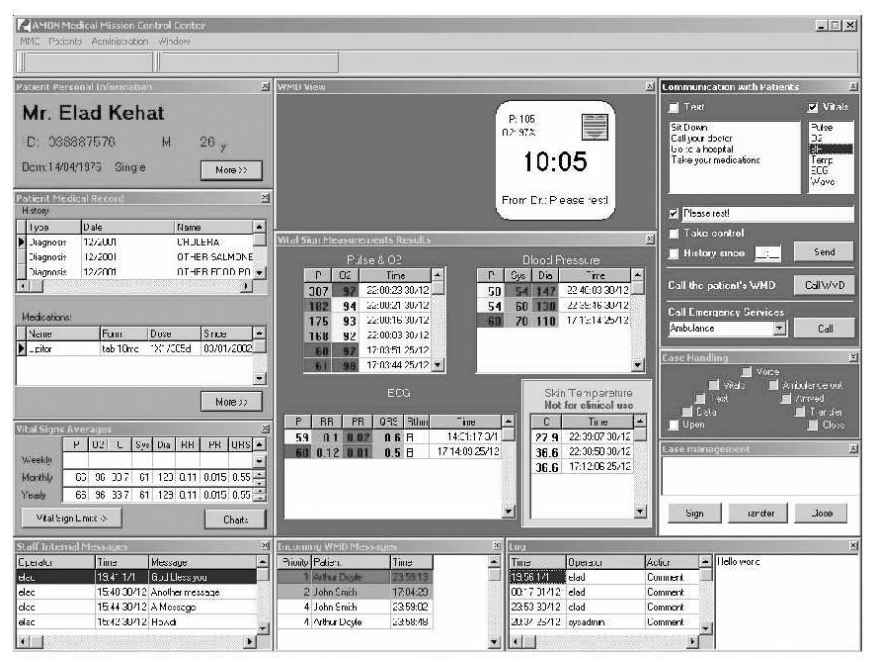

Fig. 7. Telemedicine centre screen: personal information, medical records, medications, internal messages, vital sign measurements include archived data and communication panel

on the TMC server. The server software compares the data to previous medical results in the archive and displays them in order of priority, issuing auditory alerts to the physician if necessary [Figure 7]. The physician has the ability to view the patient medical record and profile as logged on the server. The medical records summarise the medical history including diagnosis, medications and personal information such as ways to contact the patient. The purpose of this is to provide a mechanism for the consolidation of all information associated with the care and administration of a patient in a variety of environments including their home.

Within the telemedicine centre, the operational staff may triage from remote and analyse the data received from the wrist monitoring device. The support by the online telemedicine centre staff is based on expert knowledge and trend analysis of previous recorded data. For each sensor and individual parameter, an algorithm analyses the data and examines whether the parameters are within the normal medical standard limits or within predetermined values. This provides initial diagnostic, evaluation and support services for the clinicians and the user of the system. High-level algorithms analyse the multiparameter data which arrives, including the medical history and alert the medical staff accordingly.

The workstation enables:

- Remote monitoring of the AMON device

- Support and management functions such as patient diagnostic processing, clinical records review, exception condition processing and reporting

- Direct contact with the patient

- Access to the historic medical file of the patient

The medical algorithm system monitors the frequency of data input from users, screens which vital signs are more often out of range from the normal values and alerts if the values start to shift to the edge of their range over time. The staff undertake extensive accredited training for managing such a system including the legal ramifications of their role. Whilst
TABLE III

MEASURED PARAMETER RANGE AND ASSIGNED PATIENT ZONES DURING THE MEDICAL VALIDATION.

\begin{tabular}{l|c|c|} 
parameter & measurement range & patient zones (Table I) \\
\hline Systolic $(\mathrm{mmHg})$ & 104 to 150 & 29 Norm. and $4 \mathrm{H} 1$ \\
Diastolic (mmHg) & 70 to 99 & 26 Norm. and $7 \mathrm{H} 1$ \\
SpO2 (\%) & 95 to 100 & 33 Norm. \\
Pulse (bpm) & 55 to 102 & 31 Norm., 1 H1 and 1 L1 \\
QRS (s) & 0.06 to 0.18 & 32 Norm. and 1 H3
\end{tabular}

covered by best practice and current knowledge defence in cases of litigation, the final arbiter of care decisions is the clinician treating/monitoring the patient.

Only qualified operational staff (nurses, qualified physiology technicians and physicians), each with individual user name and password, have access to the medical data. All medical data is secured according to the data-protection ACT 1998, as enforced across Europe from 1 March 2000. When a medical record is open, there is a digital stamp of time, date and authorised access name. The database itself is secured on a dedicated server.

The telemedicine centre acts as a filter and monitoring service for active management of patients using the system. It is envisaged that this system will improve efficiency and effectiveness of home healthcare. The authors are aware that more advanced ways to present and preprocess the data coming in from the wrist device would enhance this effect. However, since the data is used for critical decision, any automatic preprocessing must be carefully considered. Since the focus of the project was on the mobile device such development has been postponed for further research.

\section{Medical Validation}

The goal of the medical trial is to confirm the possibility of reliably using several sensors in the same wrist-type device - without compromising on medical accuracy, as compared to standard measurement approaches. Additionally, in order to gain some feedback on user perceptions and wearability of the device, each subject was given a questionnaire to fill out.

The following parameters have been validated:

- Pulse oximeter: blood saturation in oxygen (SpO2) and pulse

- Wrist-type blood pressure monitor: systolic, diastolic, and pulse

- Three electrodes recording cardiac activity in one lead configuration: QT, QRS, and heart rate (RR interval)

The study was conducted on thirty-three healthy volunteers. The volunteers have been recruited in the SAMU 92 and the Paris Ouest medical school. Any volunteering healthy subject aged from 18 to 79, with no cardiovascular past history, nor any pulmonary disease could participate. Table III shows the measured range of the vital parameters.

The device was tested for a period of 70 minutes on each subject. The blood pressure measurement is compared to that manually measured by two trained investigators. As regards the other measurements, the AMON device is compared to other devices present on the market and fulfilling the 
TABLE IV

Grading of MEdicAl Validation. Following AAMi (AsSOCiATion FOR THE ADVANCEMENT OF MEDICAL INSTRUMENTATION) CRITERIA.

Blood pressure: 132 measurements, $87 \%(116)$ produced results

\begin{tabular}{l|c|c|c}
\hline \multirow{3}{*}{ Systolic } & $<5 \mathrm{mmHG}$ & $<10 \mathrm{mmHG}$ & $<15 \mathrm{mmHG}$ \\
Diastolic & $54 \%(62)$ & $77 \%(89)$ & $85 \%(99)$ \\
& $43 \%(50)$ & $65 \%(76)$ & $85 \%(99)$ \\
Pulse & $<5 \mathrm{bpm}$ & $<10 \mathrm{bpm}$ & $<15 \mathrm{bpm}$ \\
\hline
\end{tabular}

SpO2: 198 measurements, 68\%(135) produced results

\begin{tabular}{l|c|c|c}
\hline \multirow{2}{*}{ O2 saturation } & $\begin{array}{c}<\% \\
78 \%(105)\end{array}$ & & \\
& $<5 \mathrm{bpm}$ & $<10 \mathrm{bpm}$ & $<15 \mathrm{bpm}$ \\
Pulse & $64 \%(86)$ & $83 \%(112)$ & $89 \%(120)$ \\
\hline
\end{tabular}

ECG: 66 measurements, $84 \%(55)$ produced results

\begin{tabular}{l|c|c|c}
\hline & $<5 \mathrm{bpm}$ & $<10 \mathrm{bpm}$ & $<15 \mathrm{bpm}$ \\
Heart rate & $50 \%(27)$ & $71 \%(39)$ & $76 \%(42)$ \\
\hline
\end{tabular}

CE norms. The $\mathrm{SpO} 2$ measurement is compared to Datex cardiocap II and Nellcor NPB 40. The pulse measurement obtained by the wrist-type blood pressure monitor and the pulse oximeter is compared to the heart rate measured by the cardioscope of reference, Physiocontrol Lifepack 8. The recording of the cardiac activity is compared to the printed recording of the same period by the reference cardioscope. The comparison elements are the QT, the length of the QRS and the heart rate (RR interval). The statistical analysis is carried out separately for each sensor. The statistical method used is the Bland-Altman method [40].

\section{A. Results}

The wrist device declares that a measurement is erroneous in two situations: the wave form could not be detected, e.g. too much noise; and when the calculated parameter lies outside the valid risk zones in Table I. The assumption of the later situation is that values produced outside a valid zone are regarded as highly improbable - in particular, during the medical trials it is known that all subjects could not possibly exhibit readings outside their range. Of course in a field situation, the values would be reported back to the telemedicine centre regardless, allowing trained staff to make the decision as to whether the device is broken or something is seriously wrong with the patient.

In calculating the Medical Validation results, such erroneous results were excluded from consideration. These results are presented in Table IV, below we give some further comments to these results together with some findings from the questionnaire.

1) Measurement Results: A close investigation of the raw blood pressure data showed a design flaw in the pulse algorithm, after the correction $85 \%$ had a difference of less then 5 beats.

The deviation of the oxygen saturation measurement is far from the expected results. These results are insufficient for this system to be used with confidence in a clinical environment. The quality of the pulse measurement from the $\mathrm{SpO} 2$ sensor is, however, good.
Regarding the ECG, although heart rate results could be obtained, there is no concordance in the QRS and QT results. These poor results were found to be due to high levels of mesurement noise. With such noise on the signal, there is no possibility of successful calculations using the on-device algorithms.

2) Remarks from Questionnaire: AMON was found comfortable by $70 \% .10 \%$ - three female subjects with small wrist diameter - replied that the device was painful. The pain occurred when the blood pressure was taken.

All the subjects replied that the fact of carrying a device such as AMON would give them a feeling of security if they were suffering pathology at risk of acute complication. This sensation of security would allow them to resume their social activity and to go out. No subject has emitted a doubt about the respect of the confidentiality of the medical information for the patients being monitored by AMON. In no case the confidentiality issue would prevent them from using a device of telemonitoring with transmission of the data.

The two investigating physicians and five nurses stated that a patient presenting a chronic disease and more precisely a cardio-vascular disease could resume a somewhat autonomous life by carrying the device. However, they also stated that the design needs improvements and that the duration of measurements is too long. Additionally, it is believed that user training may be required for some of the measurements, e.g. blood pressure.

\section{B. Conclusion}

1) Measurement Results: Concerning blood pressure, the systolic pressure results are good and may lead in a future development to a final product that operates on a daily clinical basis. Diastolic measurements need to be improved and revalidated afterwards.

Measurement of blood saturation using the reflectance sensor does not provide reliable results. Nevertheless, the pulse measure using the oxygen blood saturation sensor provides good results. Detection of the pulse is a prerequisite for the measurement of blood saturation. The good measures of pulse are an encouraging aspect of this technique, which will need further development. Moreover, it is the first time the sensor is used in this configuration and in such an environment.

ECG provides poor or no results. Calculation of reliable heart rate and length of the QRS wave was not made possible. Noise was a problem for all measurements. Improvements hardware and algorithm-wise are foreseen and should improve the measurements significantly.

The results are close to what we expected but the device needs some improvements. What is sure is that the using of several sensors in the same device is possible.

2) Remarks from Questionnaire: For almost all the users (both subjects and investigators) the device has been positively rated. The answers stress the technological breakthrough and the amelioration for the patient. It can be stated from these results that there is a demand for this kind of device both from the patients and healthcare providers point of view.

An important point in all of the subjects' responses is that such a technology rich device must be totally reliable. 


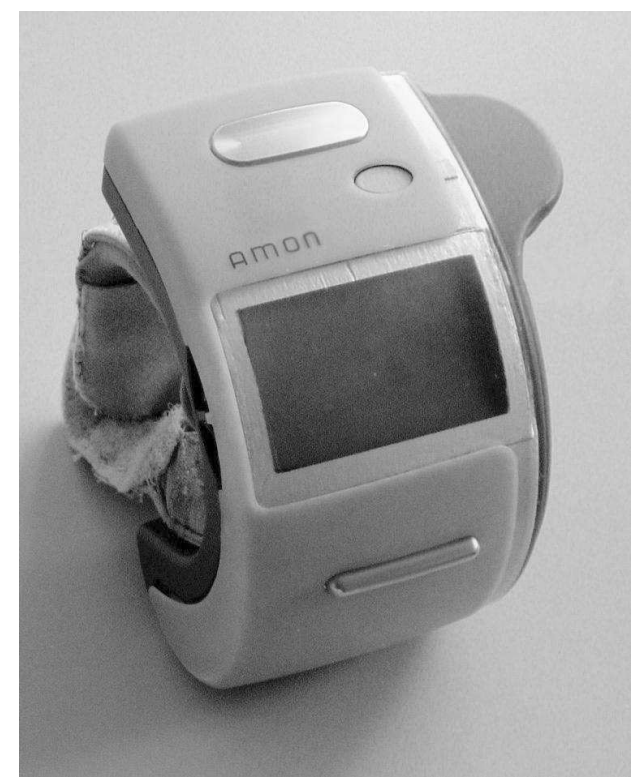

Fig. 8. AMON Prototype

A second set of remarks underlines the importance of the visual and comfort aspects of the device. It is required that the functioning of the device does not hamper the daily life of the patient. If the device increases the autonomy of the patient it must still have an aspect compatible with a daily social life. Likewise, this ties in with importance of comfort, as stressed earlier. The forecast by users of the device are very encouraging.

\section{CONCLUSION}

We have developed a wearable medical monitoring and alert system aimed at people at risk from heart and respiratory disease. The system combines multiparameter measurement of vital signs, online analysis and emergency detection, activity analysis and cellular link to a telemedicine centre in an unobtrusive wrist worn device. A prototype of both the wrist device and the medical centre software has been implemented. Medical trials were performed on 33 patients. While first prototypes had problems with achieving the required medical accuracy on all the measurement, the tests have provided a clear indication of the feasibility of the concepts and validity of the solutions adapted by the project.

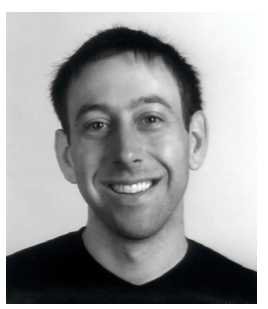

Urs Anliker received the Dipl.-Ing. (MSc) degree in electrical engineering from ETH Zurich, Switzerland, in 2000. In 2000, he joined the Wearable Computing Laboratory at the Electronics Laboratory at ETH Zurich where he is currently pursuing his $\mathrm{PhD}$. His research interests include low power wearable system design and medical systems. He is a student member of the IEEE.

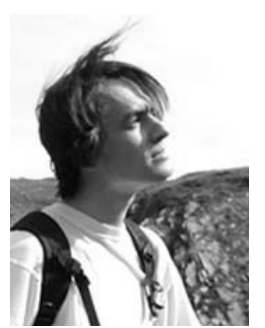

Jamie A.Ward received the BEng degree, with Honours in Computer Science and Electronics (CS\&E), from the University of Edinburgh, Scotland, in 2000. $\mathrm{He}$ spent his first year after graduation working as an analogue designer for a startup electronics firm in Austria, before joining the ETH Wearable Computing Lab in Zrich, Switzerland, where he is currently working towards his $\mathrm{PhD}$. His current research focus is on activity recognition using heterogeneous onbody sensors.

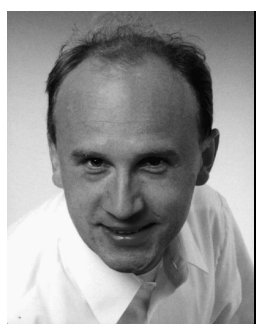

Paul Lukowicz received the MSc degree in computer science in 1992, the MSc degree in physics in 1993 , and the $\mathrm{PhD}$ degree in computer science in 1999, all from the University of Karlsruhe, Germany. Since 1999 he has been in charge of the Wearable Computing Lab and the Computer Architecture Group in the Department of Information Technology and Electrical Engineering of ETH Zurich, Switzerland. In 2003 he was appointed professor of computer science and head of the Institute for Computer Systems and Networks at the University of Health Informatics and Technology Tirol, Innsbruck, Austria. His research interests include wearable and mobile computer architecture, context and activity recognition, high performance computing, and optoelectronic interconnection technology.

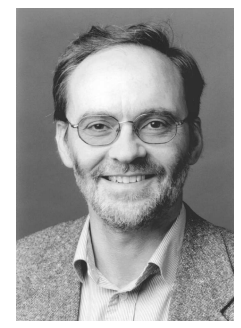

Gerhard Tröster (SM'93) received the MSc degree from the Technical University of Karlsruhe, Germany, in 1978 and the Ph.D degree from the Technical University of Darmstadt, Germany, in 1984, both in electrical engineering. He is a Professor and head of the Electronics Laboratory, ETH Zrich, Switzerland. During the eight years he spent at Telefunken Corporation, Germany, he was responsible for various national and international research projects focused on key components for ISDN and digital mobile phones. His field of research includes wearable computing, reconfigurable systems, signal processing, mechatronics, and electronic packaging. He authored and coauthored more than 100 articles and holds five patents. In 1997, he cofounded the spinoff u-blox ag.

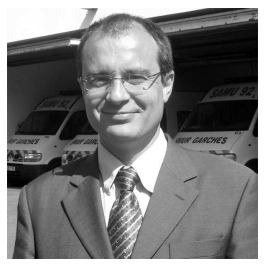

Franćois Dolveck Doctor in Médecine University of Medecine Paris 1997 Received in emergency Medecine University of Medecine Paris 1999 Received in Foreign Médecine University of Medecine Paris 2001 "Praticien Hospitalier", Emergency Medecine," Medecin des Hôpitaux" July 2003 $\mathrm{He}$ is involved in several European projects, already achieved, JUST, AMON. 


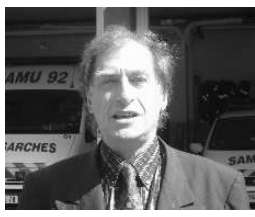

Michel Baer is a Doctor in Medicine. University of Medicine PARIS 1975 Specialist in Anesthesia and Surgical Intensive Care. PARIS, 1977 "Praticien Hospitalier", Anesthesiologist," Medecin des Hpitaux" 1984 Dr Michel Baer is the manager of the Emergency Medical Services of the Hauts de Seine department including SAMU (dispatching center) and SMUR (Intensice Care Mobile units). $\mathrm{He}$ is involved in several European projects, already achieved as HECTOR, JUST, AMON. He is the coordinator of samu.org, an EMS network of African/French EMS, financed by the "Agence intergouvernementale de la Francophonie". He is the National Coordinator for the OHEMS project (WHO), and an advisor of WHO in the field of EMS. He is the coordinator of HESCULAEP, a 4 years FP6 ERA-NET European project.

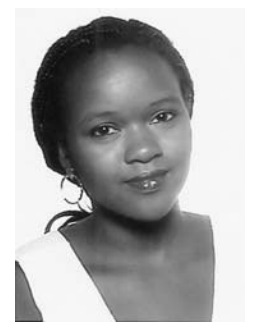

Fatou Keita text

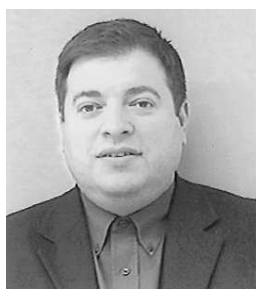

Eran B. Schenker is MedicTouch Ltd founder and Medical Director. He is a reserve commander of the first responder's unit of the Home Front Command (HFC) of the Israel Defense Forces (IDF). $\mathrm{He}$ is a graduate of the HFC Executive Search and Rescue Commanders course in 2001. He is trained to command teams in Medical emergency events, international terror events, biological and chemical warfare. He is a Medical Doctor and serves on several international medical organizations. He is founder of the Israel Aerospace Medicine Institute (IAMI) He served as the primary investigator for Israel Space Agency (ISA) bio-medical space research payload on STS-80 \& STS-95, on board the space shuttle at NASA. In 2003 he was part of the payload team of CIBX and on board Colombia space shuttle on STS107 space mission.

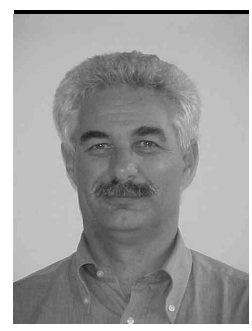

Fabrizio Catarsi received the MSc degree in Electronic Engineering in 1981, from the University of Pisa, Italy. From 1983 till 1985 he has been in charge of the development of the operating system of an automatic test equipment (ATE) at Olivetti Technost. He joined CAEN S.p.A in 1985 where he has been in the R\&D group until 1988 when he joined the Marketing division. From 1994 he has been responsible for the coordination of the $R \& D$ activities related with the development projects in the e-Health applications field. He has coordinated and still he's coordinating some research projects partially founded either from the Italian Ministry of Research or from the European Community. Since 2000 he's one of the general manager of the company.

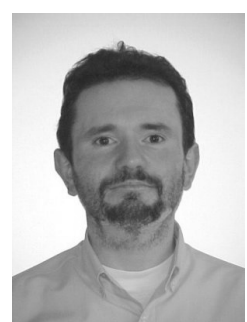

R\&D dept.
Luca Coluccini Professional qualification: Scientific High School. He has been working in Caen since 1985. He began to work in the R\&D department in the field of HV power supply. He developed CERN OBELIX Experiment High Voltage Power Supply. Then he took part in the development of the front end of Kloe Experiment. From 1991 to 1997 he had the role of R\&D manager in CAEN Mass Analysis department and designed the Mass spectrometer acquisition systems based on quadrupolar mass filter. Since 1997 he is Senior Engineer in CAEN e-healthy

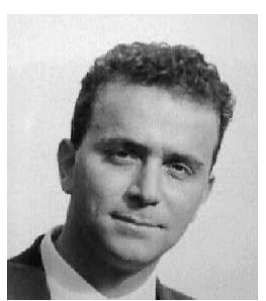

Andrea Belardinelli Text

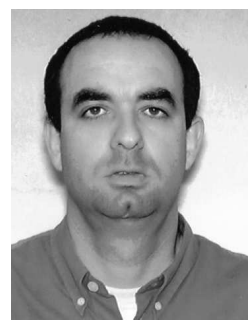

Dror Shklarski received the BSc. in Electronics and Electrical Engineering, Cum Laude, Tel-Aviv University, in 1991 and the MSc in Management and Industrial Engineering, Ben-Gurion University, Israel, in 1996. From 1991-1997 he was Project Manager for three major communication programs in Israeli Air-Force. Since 1998, he works in Tadiran Spectralink. In 1998-2001 he was Program Manager for Wireless Communications Systems program. From 2001 he has been Senior Director of the civilian data link R\&D branch of Tadiran Spectralink and responsible for the telehealth projects of Tadiran Spectralink.

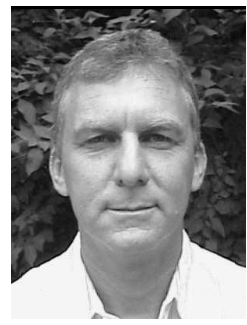

Menachem Alon received the BSc degree in electrical engineering from the Technion Institute Israel in 1986. From 1986 to 2001 he was working for RAFAEL Israel - Avionic Projects Directorate as a system engineer. In 2001 he joined Tadiran Spectralink as a technical manager responsible for all R\&D activities focused mainly on cellular data links for embedded devices and avionic multifunction control units.

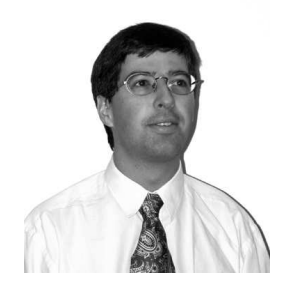

Etienne Hirt received the M.Sc. degree in electrical engineering and the Ph.D. degree from the Swiss Federal Institute of Technology (ETH), Zrich, in 1995 and 2000, respectively (ISBN 3-89649-5593). He joined the Electronics Laboratory, ETH, in Spring 1995 as a Teaching and Research Assistant in the Multichip Module Group. He worked in the fields of system and package design methodology using multichip modules and high density packaging technologies. He jointly developed two Pentium based multichip modules and an FPGA based MCM. In 1999 Etienne Hirt co-founded the company Art of Technology AG, which specializes in product engineering using high density packaging. He is member of the management as director $\mathrm{R} \& \mathrm{D}$. 


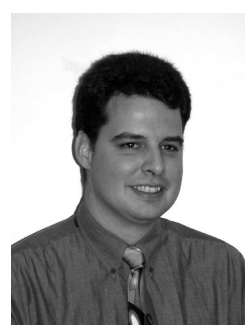

Rolf Schmid Born in 1969, Rolf Schmid received his Master Degree in Electrical Engineering from the Swiss Federal Institute of Technology of Zurich (ETH Zrich) in 1996. Until 2002 he worked as a scientific employee at the Electronics Lab of ETH, responsible for the EC project EUROPRACTICE that had the focus on dissemination of HDP/MCMtechnologies throughout Europe. As a co-founder of the Art of Technology AG he is heading the company as Managing Director since 1999.

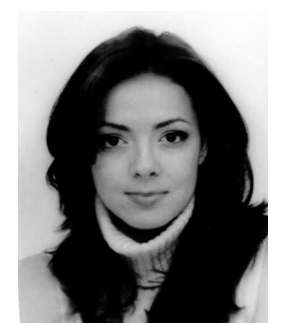

Milica Vuskovic was born in 1976 in Belgrade, Serbia and Montenegro. She received the Master Degree in Electrical Engineering from the Swiss Federal Institute of Technology of Zurich (ETH Zrich) in 2001. During 2002 she developed power supply and control systems for automotive applications as a research assistant at Measurement and Control Laboratory at ETH Zrich. She is currently employed by Art of Technology in Zurich, where she is working on several engineering fields, including power supply for wireless devices, automotive systems, and FPGA and analog and digital hardware design.

\section{ACKNOWLEDGMENT}

The AMON project has been funded by the EU IST FP5 program. The enclosure production and design has been perfomed by Estragon AG, Switzerland.

\section{REFERENCES}

[1] S. Mann. Wearable computing as means for personal empowerment. In Proc. 3rd Int. Conf. on Wearable Computing (ICWC), May 1998.

[2] T. Starner. The challenges of wearable computing: Part 1. IEEE Micro, 21(4):44-52, July 2001.

[3] A. Belardinelli, G. Palagi, R. Bedini, A. Ripoli, V. Macellari, and D. Franchi. Advanced technology for personal biomedical signal logging and monitoring. In Proc. of the 20th Annual International Conference of the IEEE Engineering in Medicine and Biology Society, volume 3, pages 1295-1298, 1998.

[4] E. Jovanov, T. Martin, and D. Raskovic. Issues in wearable computing for medical monitoring applications: A case study of a wearable ecg monitoring device. In The Fourth International Symposium on Wearable Computers (ISWC), pages 43-49, Oct. 2000.

[5] Omron corporation. http://www.omron.com.

[6] Polar heart rate monitors. http://www.polar.fi/.

[7] Bodymedia, inc. http://www.bodymedia.com/.

[8] E. Jovanov, P. Gelabert, B. Wheelock, R. Adhami, and P. Smith. Real time portable heart monitoring using low power dsp. In International Conference on Signal Processing Applications and Technology ICSPAT, Oct. 2000

[9] E. Jovanov, D. Raskovic, J. Price, A. Moore, J. Chapman, and A. Krishnamurthy. Patient monitoring using personal area networks of wireless intelligent sensors. Biomedical Sciences Instrumentation, 37:373-378, 2001.

[10] Escort guardian, invivo research, inc. http://www.invivoresearch.com/.

[11] Micropaq, wellch allyn, inc. http://www.monitoring.welchallyn.com/.

[12] S. Pavlopoulos, E. Kyriacou, A. Berler, S. Dembeyiotis, and D. Koutsouris. A novel emergency telemedicine system based on wireless communication technology ambulance. IEEE Transaction on Information Technology in Biomedicine, 2(4):261-267, December 1998.

[13] S.L. Toral, J.M. Quero, M. Elna Pérez, and L.G. Franquelo. A microprocessor based system for ecg telemedicine and telecare. In The 2001 IEEE International Symposium on Circuits and Systems, volume IV, pages 526-529, 2001.

[14] Jing Bai, Yonghong Zhang, Delin Shen, Lingfeng Wen, Chuxiong Ding, Zijing Cui, Fenghua Tian, Bo Yu, Bing Dai, and Jupeng Zhang. A portable ecg and blood pressure telemonitoring system. IEEE Engineering in Medicine and Biology, pages 63-70, July/August 1999.

[15] E. Jovanov, A. O'Donnel Lords, D. Raskovic, P.G. Cox, R. Adhami, and F. Andrasik. Stress monitoring using distributed wireless intelligent sensor system. IEEE Engineering in Medicine and Biology Magazine, pages 49-55, May/June 2003.

[16] M. Akay, M. Sekine, T. Tamura, Y. Higashi, and T. Fujimoto. Unconstrained monitoring of body motion during walking. IEEE Engineering in Medicine and Biology Magazine, pages 104-109, May/June 2003.

[17] Y. Mendelson and B.D. Ochs. Noninvasive pulse oximetry utilizing skin reflectance photoplethysmography. IEEE Transactions on Biomedical Engineering, 35(10):798 -805, Oct. 1988.

[18] M. Nogawa, T. Kaiwa, and S. Takatani. A novel hybrid reflectance pulse oximeter sensor with improved linearity and general applicability to various portions of the body. In Proceedings of the 20th Annual International Conference of the IEEE, volume 4, pages $1858-1861$, Oct./Nov. 1998.

[19] Spo medical equipment ltd. http://www.spomedical.com/.

[20] B. B. Winter and J. G. Webster. Driven-right-leg circuit design. IEEE Transaction Biomedical Engineering, 30(1):62-66, 1983.

[21] J.J. Carr and J. M. Brown. Introduction to Biomedical Equipment Technology. Prentice Hall, Inc., 3rd edition, 1998.

[22] K.F.Tan, K.L.Chan, and K.Choi. Detection of the qrs complex, p wave and $\mathrm{t}$ wave in electrocardiogram. In Proceedings of International Conference on Advances in Medical Signal and Information Processing, pages 41-47, Sept. 2000.

[23] J. Pan and W. J. Tompkins. A real-time qrs detection algorithm. IEEE Trans. Biomed. Eng., pages BME-32 (3):230-236, 1985.

[24] M. Ursino and C. Cristalli. A mathematical study of some biomechanical factors affecting the oscillometric blood pressure measurement. IEEE Transaction on Biomedical Engineering, 43(8):761-778, August 1996.

[25] JCTB moraes, M. Cerulli, and PS. Ng. Development of a new oscillometric blood pressure measurement system. In Computers in Cardiology, volume 26, pages 467-470, 1999.

[26] Drzewiecki et al. Theory of the oscillometric maximum and the systolic and diastolic detection ratios. Annals of Biomedical Engineering, 22:8896, 1994. 
[27] J. Farringdon, A.J. Moorea, N. Tilbury, J. Church, and P.D. Biemond. Wearable sensor badge and sensor jacket for context awareness. In 3rd International Symposium on Wearable Computers (ISWC), pages 107113, 1999.

[28] G. Williams, K. Doughty, K. Cameron, and D.A. Bradley. A smart fall and activity monitor for telecare applications. In Proceedings of the 20th Annual International Conference of the IEEE Engineering in Medicine and Biology Society, volume 20, 1998.

[29] T. Degen, H. Jaeckel, M. Rufer, and S. Wyss. Speedy: A fall detector in a wristwatch. In 7th International Symposium on Wearable Computers (ISWC), Oct. 2003.

[30] N. Kern, B. Schiele, H. Junker, and P. Lukowicz. Wearable sensing to annotate meeting recordings. In 6th International Symposium on Wearable Computers (ISWC), Oct 2002.

[31] G.L. Brengelmann. Body surface temperature: Manifestation of complex anatomy and physiology of the cutaneous vasculature. In Proceedings of the 22nd Annual EMBS International Conference, pages 1927-1930, July 2000.

[32] World health organisation. http://www.who.int/.

[33] L. Benini and G. De Micheli. System-level power optimization: Techniques and tools. In ISLPED99: International Symposium on Low Power Electronics and Design, pages 288-293, august 1999.

[34] J. Pouwelse, K. Langendoen, and H. Sips. Dynamic voltage scaling on a low-power microprocessor. In Proc. 7th ACM Int. Conf. on Mobile Computing and Networking (Mobicom), pages 251-259, Rome, Italy, july 2001.

[35] Y. Li, Y. Zha, M. Oberle, and A. Deiss. Integrated frontend for electrocardiography (ecg). In Student Thesis,Integrated Systems Laboratory, ETH Zürich, 2000.

[36] T. Ishihara and H. Yasuura. Voltage scheduling problem for dynamically variable voltage processors. In Proc. Int'l Symposium on Low Power Electronics and Design, pages 197-202, 1998.

[37] European directive 95/46/ec of the european parliament and of the council of 24 october 1995 on the protection of individuals with regard to the processing of personal data and on the free movement of such data. In Official Journal L.281, 23 November 1995.

[38] M.I. Silventoinen and T. Rantalainen. Mobile station emergency locating in gsm. In IEEE International Conference on Personal Wireless Communications, pages 232-238, Feb 1996.

[39] C. Drane, M. Macnaughtan, and C. Scott. Positioning gsm telephones. IEEE Communications Magazine, pages 46-59, April 1998.

[40] J. M. Bland and D. G. Altman. Statistical methods for assessing agreement between two methods of clinical measurement. In Lancet, volume i, pages 307-310, 1986. 